\title{
Habilidades verbais e recursos imagéticos na coerência narrativa de eventos autobiográficos ${ }^{1}$
}

\author{
Luciano da Silva Alencastro ${ }^{2}$ \\ Luciano Ferreira Piccoli \\ William Barbosa Gomes \\ Universidade Federal do Rio Grande do Sul, Porto Alegre-RS, Brasil
}

\begin{abstract}
Resumo: A qualidade da narrativa autobiográfica parece depender tanto de habilidades verbais (estilo e coerência), quanto de recursos imagéticos. No entanto, não se sabe qual o impacto de estilos cognitivos verbal/visual na evocação das lembranças que compõem a narrativa. Oitenta e dois estudantes universitários dos cursos de psicologia, letras, biomedicina e odontologia, com idades entre 17 e 54 anos (média $=22,66$ anos; $D P=6,2$ ), relataram um evento autobiográfico e responderam a questionários sobre memória autobiográfica, estilos cognitivos visual-verbal, e imaginação. Os resultados não indicaram a presença de referências visuais na narrativa autobiográfica, mas apontaram para diferenças quanto a estilo verbal, número de palavras e coerência narrativa entre grupos de estudantes (psicologia e letras versus odontologia e biomedicina). A interação entre as três variáveis foi sustentada pela correlação entre coerência narrativa e número de palavras $(r=0,769 ; p=0,001)$, e pelos diferentes perfis narrativos entre cursos universitários.
\end{abstract}

Palavras-chave: narrativas, memória autobiográfica, estilo cognitivo.

\section{Verbal skills and imagistic resources in the narrative coherence of autobiographical events}

\begin{abstract}
The quality of an autobiographical narrative seems to depend on both verbal skills (style and coherence) and imagistic resources. However, the impact of cognitive verbal and/or visual styles on the evocation of memories that produce the narrative is still unclear. A total of 82 college students from different fields (Psychology, Languages, Biomedicine and Dentistry), aged between 17 and 54 years old (mean $=22.66$ years, $S D=6.2$ ) wrote an autobiographical event and answered to three questionnaires: autobiographical memory, verbalizer-visualizer, and imagination. The results did not indicate the presence of visual cues in the autobiographical narrative, but showed differences between two groups of students (Psychology and Language versus Dentistry and Biomedicine) for verbal style, number of words and narrative coherence. The interaction among these three variables was supported by both the correlation between narrative coherence and number of words $(r=0.769, p=$ $0.001)$, and by the narrative profiles among college groups.
\end{abstract}

Keywords: narratives, autobiographical memory, cognitive style.

\section{Habilidades verbales y recursos de imaginería en la coherencia narrativa de eventos autobiográficos}

\begin{abstract}
Resumen: La calidad de la narración autobiográfica parece depender de las habilidades verbales y de los recursos de imaginería. Sin embargo, no está claro el impacto de los estilos cognitivos verbales-visuales en los recuerdos que componen la narración. Ochenta y dos estudiantes de Psicología, Letras, Biomedicina y Odontología desde 17 hacia 54 años (promedio = 22,66 años, $D E=6,2$ ) escribieron un relato autobiográfico y respondieron a tres cuestionarios: memoria autobiográfica, estilos cognitivos verbalizador-visualizador, y imaginación. Los resultados no indicaron la presencia de pistas visuales en la narrativa autobiográfica, pero evidenciaron diferencias entre los dos grupos de alumnos (Psicología y Letras ante Odontología y Biomedicina) relacionadas a estilo verbal, número de palabras y coherencia narrativa. La interacción entre estas tres variables fue apoyada por la correlación entre la coherencia narrativa y el número de palabras $(r=0,769, p=0,001)$, y los perfiles de narrativa entre los grupos de estudiantes.
\end{abstract}

Palabras clave: narrativas, memoria autobiográfica, estilo cognitivo.

Memórias autobiográficas constituem-se de registros duradouros, lembrados em longo prazo (Westbury \&

\footnotetext{
${ }^{1}$ Este texto foi revisado seguindo o Acordo Ortográfico da Língua Portuguesa (1990), em vigor a partir de $1^{\circ}$ de janeiro de 2009. Este trabalho contou com o apoio do CNPq.

${ }^{2}$ Endereço para correspondência:

Luciano da Silva Alencastro. Universidade Federal do Rio Grande do Sul. Instituto de Psicologia. Rua Ramiro Barcelos, 2600/sala 123. CEP 90.035-003. Porto Alegre-RS, Brasil.E-mail: lu.alen@ibest.com.br.
}

Dennett, 2000), sendo revividas com qualidades quase sensoriais e com forte senso de veracidade (Conway, 2001). Eventos que ocasionam tais memórias são lembrados com facilidade, de forma vívida e em detalhes e se tornam mar$\cos$ na organização da trajetória individual (Elnick, Margrett, Fitzgerald, \& Labouvie-Vief, 1999) e na compreensão do sentido da própria história de vida. Por conseguinte, as experiências de vida aparecem organizadas em enredos de eventos sucessivos expressos na forma de narrativas. 


\section{Coerência narrativa autobiográfica e recursos verbais-imagéticos}

Narrativas autobiográficas se caracterizam pela maior ou menor coerência de seus conteúdos e articulações, e podem ser diferenciadas quanto: (1) à coerência temporal, indicada pela habilidade de organizar os eventos de forma cronológica; (2) à coerência temática, indicada pela habilidade de situar adequadamente os fatos ocorridos e de estabelecer relações de similaridade entre eles; (3) à coerência causal, indicada pela habilidade de situar os antecedentes e os consequentes que permeiam os eventos pessoais (Habermas \& Bluck, 2000).

A qualidade da narrativa de uma recordação autobiográfica depende da sua forma de organização, isto é, da estrutura e coerência (Rubin \& Greenberg, 2003), e do modo como utiliza recursos ilustrativos ou imagéticos (Rubin, Schrauf, \& Greenberg, 2003). Rubin (1998) ressaltou que a imagem é um poderoso recurso facilitador para a recordação, aumentando a especificidade e a autorreferência nos eventos pessoais. Segundo o autor, detalhes específicos fornecidos pela imagem mental fariam com que as recordações parecessem mais precisas e passíveis de serem verdadeiras. Por conseguinte, o indivíduo acreditaria que não estava apenas imaginando, mas revivendo o evento. O papel da imageabilidade visual na memória autobiográfica tem sido demonstrado em vários estudos empíricos (Knez, 2006; Rubin, 2005). Contudo, tem sido difícil diferenciar o que é visual e verbal na recordação autobiográfica.

\section{Narrativa autobiográfica e estilos cognitivos}

Influências imagéticas e/ou verbais na recordação e na narrativa autobiográfica podem estar associadas ao estilo cognitivo, isto é, ao modo preferencial na utilização de recursos cognitivos como pensar, perceber, lembrar e resolver problemas (Martins, Santos, \& Bariani, 2005). Não se pode, em princípio, distinguir bons e maus estilos. Eles são apenas diferentes.

A hipótese dos estilos cognitivos vem sendo estudada em psicologia da educação (Felder \& Spurlin, 2005; Ormrod, 2008) e das organizações (Cools \& Van den Broeck, 2007). Ormrod é um dos defensores desta hipótese, referindo que alguns alunos parecem aprender melhor quando a informação é apresentada por meio de palavras (estilo cognitivo verbal), enquanto outros parecem aprender melhor quando a informação é apresentada por meio de imagens (estilo cognitivo visual).

Apesar do escopo do presente estudo concentrar-se tanto em diferenças verbais quanto imagéticas, é importante situar a existência de outras dimensões conceituais dos estilos cognitivos, tais como: impulsividade versus reflexividade, pensamento convergente versus divergente, e pensamento holista versus serialista. Pessoas com estilo cognitivo holista, por exemplo, utilizariam uma estratégia global para a resolução de problemas, abordando simultaneamente as várias partes da tarefa. Pessoas com estilo cognitivo serialista utilizariam uma estratégia local, abordando um aspecto do problema por vez (Cegarra \& Hoc, 2006).

O trabalho de Bruner, Goodnow e Austin (1956) tem sido apontado como pioneiro no reconhecimento de preferências cognitivas no aprendizado. Neste estudo, os autores também investigaram como se dá a construção de conceitos, o que posteriormente levou Bruner (1986/1997) a constatar a existência de duas lógicas de pensamento: o pensamento paradigmático e o pensamento narrativo. Apesar de Bruner não denominar estas diferentes lógicas como preferências cognitivas, esta vertente de teorização serviu de inspiração para que, mais tarde, outros estudiosos postulassem as diferentes dimensões de estilo cognitivo - como o pensamento holista versus serialista (Cegarra \& Hoc, 2006).

Bruner situa o pensamento paradigmático e o pensamento narrativo dentro da seguinte polaridade: abstração de particularidades (paradigmático) versus descrição detalhada de particularidades (narrativo). O pensamento paradigmático comprova a realidade via estabelecimento de provas formais e empíricas. O pensamento narrativo não busca a certeza, mas a verossimilhança. $\mathrm{O}$ primeiro leva à busca de verdades universais, o segundo à compreensão de condições particulares prováveis entre dois eventos. $\mathrm{O}$ pensamento paradigmático tenta construir um sistema formal e matemático de descrição e explicação. O pensamento narrativo, por sua vez, vincula-se à imaginação, conduzindo à construção de histórias envolventes e que abarcam a intencionalidade humana.

\section{Estilos cognitivos e diferenças entre cursos universitários}

Uma das principais manifestações e afirmações de estilo cognitivo apresenta-se na escolha de cursos universitários (Mastor \& Ismail, 2004). Muitos pesquisadores (Jones, Reichard, \& Mokhtari, 2003; Mastor \& Ismail, 2004) têm ressaltado que um dos principais fatores determinantes do desempenho acadêmico é a adequação entre o estilo cognitivo individual e as habilidades cognitivas necessárias a cada curso.

As diferenças de estilos cognitivos entre cursos universitários se encontram amplamente documentadas na literatura. Jones e cols., (2003) investigaram se as preferências cognitivas de 105 estudantes universitários variavam de acordo com o curso (inglês, matemática, ciências e estudos sociais). Os autores utilizaram um instrumento (Kolb Learning Style Inventory) que avaliava os seguintes estilos: experimentação concreta, observação reflexiva, conceitualização abstrata e experimentação ativa. Foram encontradas diferenças significativas quanto ao curso.

A investigação das preferências de aprendizagem entre alunos de psicologia constatou alto interesse por áreas verbais, subjetivas e narrativas, contrastando com um baixo interesse em atividades abstratas, como cálculo (Bueno, Lemos, \& Tomé, 2004; Levenfus, 2002). Estudantes de medicina, por sua vez, costumam apresentar estilo cognitivo com forte 
predominância de conceituação abstrata com vistas a respostas pontuais e específicas (Sobral, 2005).

Apesar das evidências obtidas em nível cognitivo (Kozhevnikov, 2007; Sternberg, Grigorenko, \& Zhang, 2008; Ruttun, 2009), bem como em nível neural (Kraemer, Rosenberg, \& Thompson-Schill, 2009), não há consenso acerca da consistência empírica do construto estilo cognitivo. As evidências obtidas ainda não são conclusivas, dada a diversidade de definições teóricas, a inconsistência psicométrica de muitos instrumentos e a fragilidade dos delineamentos metodológicos utilizados neste campo de pesquisa (Massa \& Mayer, 2006; Pashler, McDaniel, Rohrer, \& Bjork, 2008). Ciente destas controvérsias, o presente estudo propõe-se a contribuir na investigação de possíveis diferenças verbais e imagéticas na recordação e narrativa autobiográfica de estudantes universitários.

Desta forma, o objetivo deste estudo é diferenciar manifestações verbais e imagéticas na narrativa de eventos autobiográficos (memória autobiográfica) e nos estilos cognitivos que caracterizam o processo de recordação. Primeiro, examina quais as relações entre: (1) características de memória autobiográfica; (2) estilo cognitivo verbal; (3) habilidades de imaginação visual e auditiva; e (4) coerência da narrativa do evento autobiográfico. A seguir, concentra-se na verificação de diferenças de estilos cognitivos enquanto preferências verbais em narrativas autobiográficas entre estudantes universitários de diferentes áreas de conhecimento.

Esperava-se: (1) que a recordação de elementos visuais do evento estivesse associada à expressão de detalhes visuais no relato autobiográfico; (2) que a recordação vívida de imagens (tanto na recordação quanto no relato verbal do evento) estivesse associada a altos escores de imaginação visual; (3) que a recordação dos elementos ideacionais e verbais do evento (tanto na recordação quanto no relato verbal do evento) estivesse associada a altos escores em estilo cognitivo verbal; (4) que a recordação avaliada como coerente estivesse associada à coerência narrativa do evento autobiográfico; e (5) que houvesse diferenças quanto às manifestações verbais e narrativas de alunos de diversos cursos universitários.

\section{Método}

\section{Participantes}

Participaram deste estudo 82 estudantes universitários, sendo 58 mulheres e 24 homens entre 17 a 54 anos (média $=22,66$ anos; $D P=6,2$ ), matriculados em uma universidade pública da região sul. Os estudantes cursavam psicologia $(n=21)$, letras $(n=22)$, biomedicina $(n=19) \mathrm{e}$ odontologia $(n=20)$.

\section{Instrumentos}

Foram utilizados: (1) Protocolo para relato escrito de eventos autobiográficos, (2) Questionário de Memória
Autobiográfica, (3) Questionário Verbalizador-visualizador, (4) Questionário Bett's de Imaginação.

Protocolo para relato escrito de eventos autobiográficos (Santos \& Gomes, 1997): solicita-se aos participantes para recordar um evento marcante em suas vidas, dar um título a esse evento, e o relatar por escrito em uma folha de papel pautado tamanho A4. Em geral, essa simples solicitação enseja a preparação de uma breve narrativa na qual podem ser observadas características da recordação autobiográfica e de estilo de expressão narrativa.

Questionário de Memória Autobiográfica (QMA) (Rubin e cols., 2003): consiste em 21 afirmativas sobre qualidades fenomenais (como o senso de reviver o episódio), processos cognitivos (como a lembrança do evento em forma de palavras e imagens) e propriedades atribuídas a eventos pessoais (como a avaliação sobre a importância de um evento). Os itens são seguidos de uma escala Likert de 1 (não consigo recordar) a 7 (recordo completamente). O instrumento foi traduzido para o português e adaptado por Gauer (2005). Em estudo com grupo de 208 universitários brasileiros, Gauer e Gomes (2008) encontraram uma solução de dois fatores principais para o instrumento. No fator 1 permaneceram todos os itens correspondentes às qualidades fenomenais da recordação (como o senso de revivência e a crença na realidade do evento); às modalidades sensoriais da imaginação (visual, auditiva e espacial); e itens ligados à coerência do evento recordado. $\mathrm{O}$ fator 2 reuniu os itens relacionados às avaliações que se faz sobre o evento e não mais ao ato de lembrar dele, tais como a importância do evento e o quanto ele foi incomum. Como exemplo de itens, podem ser citados: "lembrando o episódio, eu posso vê-lo na minha mente"; "lembrando o episódio, eu posso lembrar o cenário onde ocorreu"; "lembrando o episódio, ele me vem em palavras". O questionário foi desenvolvido como um artifício operacional para fazer as pessoas voltarem a atenção para a experiência de estar tendo uma memória. Sendo assim, o QMA não avalia desempenho, traço ou estados de personalidade. Sua intenção não é reduzir as variáveis a um escore e sim levantar qualidades descritivas do ato de recordar.

Questionário Verbalizador-visualizador (QVV) (Richardson, 1977): avalia a predominância verbal ou visual no estilo cognitivo do indivíduo através de 30 itens de escolha forçada entre as respostas "verdadeiro" e "falso". No entanto, a consistência interna para esse formato foi baixa com alfa de Cronbach 0,30 (Pérez-Fabello \& Campos, 2004). Neste estudo, os itens estão seguidos de uma escala Likert de 1 (discordo totalmente desta afirmativa) a 7 (estou totalmente de acordo com a afirmativa), já que esta alteração aumentou a consistência interna do instrumento com alfa de Cronbach para 0,71 (Mayer \& Massa, 2003). No instrumento original, dez itens avaliavam estilo cognitivo visual (como os itens "não consigo gerar uma imagem mental do rosto de um amigo quando fecho os olhos" e "quando leio um romance consigo ter uma imagem mental da cena que é descrita"), dez itens avaliavam estilo cognitivo verbal (como os itens "tenho 
maior facilidade com as palavras do que a média" e "tenho prazer em trabalhos que requerem o uso de palavras") e dez itens funcionavam como distratores (como o item "por mais que tente me concentrar, aparecem pensamentos que não são relacionados ao meu trabalho").

Em estudo com um grupo de 104 universitários brasileiros, Alencastro, Piccoli e Gomes (2010) encontraram uma solução de 3 fatores principais para o instrumento. $\mathrm{O}$ fator 1 apresentou alfa de Cronbach de 0,185, o fator 2 alfa de 0,703 e o fator 3 alfa de 0,555 . O fator 2 - que agrupou cinco itens referentes a estilo cognitivo verbal - foi o único que apresentou boas propriedades psicométricas, indicando que o instrumento parece ser adequado apenas para mensuração de estilo cognitivo verbal. Desta forma, o presente estudo utilizou este fator a fim de avaliar estilo verbal. A pontuação do fator verbal vai de 5 a 35 pontos (cinco itens em escala Likert de 1 a 7), e a do fator visual vai de 10 a 70 pontos (dez itens do agrupamento visual da versão original do instrumento seguidos de escala Likert de 1 a 7). Na tradução do inglês para o português, a escala passou por avaliação de cincos professores de língua inglesa para ajustes de correspondência semântica.

Questionário Bett's de Imaginação (Sheehan, 1967): avalia a vivacidade da imageabilidade em sete modalidades sensórias: visual, auditiva, tátil, sinestésica, gustativa, olfativa e orgânica. A versão aqui utilizada - submetida ao mesmo processo de tradução e adaptação do QVV - avaliou apenas as modalidades visual e auditiva. Esta escolha baseia-se na hipótese de que estas dimensões podem estar relacionadas aos itens do QMA que avaliam se o evento vem em imagens, palavras, história coerente e sons. Cada uma das duas modalidades tem 5 itens, seguidas de uma escala de 1 (não consigo formar nenhuma imagem em minha mente) a 7 (imagino de forma perfeitamente clara e tão vívida quanto a experiência real). Desta forma, a pontuação para cada uma das modalidades sensórias vai de 5 a 35 pontos. Como exemplo de item pode ser citado: "pense em algum parente ou amigo que você vê frequentemente, considerando cuidadosamente a imagem que aparece em sua mente. Classifique as imagens de acordo com as seguintes questões e conforme o grau de clareza e vivacidade: o exato contorno da face, cabeça, ombros e corpo (1 a 7)". O instrumento apresenta alta consistência interna: 0,92 (Campos \& Pérez-Fabello, 2005); 0,91 (Westcott \& Rosenstock, 1976), 0,78 (Sheehan, 1967).

\section{Procedimento}

\section{Coleta de dados}

Os estudantes foram convidados, em sala de aula, para participar de uma pesquisa sobre memória, estilos de aprendizagem e narrativa. Os instrumentos foram aplicados na seguinte ordem: Protocolo de Eventos Marcantes, Questionário de Memória Autobiográfica, Questionário Verbalizador-visualizador e Questionário Bett's de Imaginação. Os pesquisadores forneciam informações sobre os instrumentos, explicando o funcionamento das escalas, lendo os dois primeiros itens dos questionários e esclarecendo eventuais dúvidas.

\section{Análise dos dados}

Inicialmente foram realizadas duas análises para verificar a coerência narrativa dos relatos autobiográficos. A primeira análise seguiu o modelo utilizado por Peterson e Biggs (1998), em que a coerência narrativa é avaliada com base em três categorias mutuamente exclusivas: orientações, ações e avaliações. O relato foi dividido em proposições que foram classificadas de acordo com as três categorias. Nesse modelo: (1) as orientações fornecem informações sobre o contexto do evento recordado (localização espacial e temporal, pessoas presentes etc.); (2) as ações trazem a factualidade do evento; (3) as avaliações referem-se aos sentimentos e pensamentos a respeito do evento recordado. Foram calculados três índices por meio da divisão do número de proposições para cada categoria pelo total de proposições no relato. Por fim, as proporções de proposições em cada categoria foram transformadas em porcentagens e denominadas de coerência narrativa.

A segunda análise utilizou uma escala de coerência narrativa global adaptada de Bohn e Berntsen (2008). A escala original era de 0 a 3 , em que 0 correspondia a um padrão desorientado (a narrativa é tão confusa que não permite uma compreensão adequada); 1 correspondia a um padrão cronológico (a narrativa é uma simples descrição de eventos sucessivos, no estilo “e então... e então..."); 2 correspondia a um padrão cronológico interessante (a narrativa descreve eventos sucessivos em detalhes e com uso de adjetivos, advérbios e conectivos entre as sentenças); e 3 correspondia a um padrão clássico (a narrativa inicia, progride até o clímax, depois do qual ocorre um desfecho). No decorrer do estudo, os referidos autores optaram por reduzir a escala, deixando de utilizar a categoria 3, já que sua amostra - crianças e jovens entre 9 e 15 - não apresentou relatos com um padrão clássico. Para o presente estudo ampliou-se a escala para abarcar a diversidade e a complexidade narrativa encontrada nos relatos de estudantes universitários com idade média de 23,3 anos. A escala utilizada, baseada nas sugestões de Habermas e Bluck (2000), foi a seguinte: 0 - padrão desorientado; 1 - a narrativa possui elementos fragmentados de orientação espaço-temporal, contexto temático e significado do evento; 2 - padrão cronológico; 3 - padrão cronológico com grande quantidade de avaliações subjetivas; 4 - narrativa situa o evento no tempo e no espaço, utilizando de forma moderada adjetivos, advérbios e conectivos entre as sentenças; 5 - narrativa cronologicamente interessante, rica em adjetivos, advérbios e conectivos; 6 - padrão clássico (a narrativa inicia, progride até o clímax, depois do qual ocorre um desfecho; toda narração denota destacado apuro no uso de adjetivos, advérbios e 
conectivos). Dois juízes codificaram independentemente $60 \%$ dos relatos (categorização quanto aos três tipos de proposição e quanto à escala de coerência global), atingindo $85 \%$ de concordância. Discordâncias foram resolvidas por discussões. O restante dos relatos foi codificado por um dos juízes (segundo autor).

A Tabela 1 descreve as médias e desvios-padrão dos escores de coerência global, do número de proposições para cada categoria de coerência microestrutural (orientações, ações e avaliações), bem como dos escores dos fatores que avaliam: (1) estilos cognitivos verbal e visual (QVV), (2) imaginação visual e auditiva (Questionário Bett's). Conforme detalhado na seção Método, a pontuação do fator verbal do QVV vai de 5 a 35 pontos, e a do fator visual vai de 10 a 70 pontos. A pontuação para cada uma das modalidades sensórias do Questionário Bett's vai de 5 a 35 pontos.

Tabela 1

Médias e desvios-padrão das variáveis investigadas

\begin{tabular}{lcccc}
\hline & Mínimo & Máximo & Média & $D P$ \\
\hline Verbal QVV* & 13 & 33 & 23,90 & 4,29 \\
Visual QVV & 30 & 65 & 49,50 & 6,574 \\
Visual Betts & 10 & 35 & 28,22 & 4,77 \\
Auditivo Betts & 13 & 35 & 26,30 & 5,23 \\
Total Betts & 24 & 70 & 54,52 & 8,76 \\
Coerência Global & 0 & 6 & 2,74 & 1,51 \\
Coerência Narrativa & & & & \\
$\quad$ Orientações & 0 & 100 & 41,09 & 17,58 \\
Ações & 0 & 50 & 23,61 & 12,94 \\
Avaliações & 0 & 86 & 35,44 & 19,29 \\
No de palavras & 3 & 279 & 105,61 & 63,85 \\
\hline
\end{tabular}

* Questionário Verbalizador-visualizador

\section{Considerações éticas}

O estudo foi aprovado pelo Comitê de Ética do Instituto de Psicologia da Universidade Federal do Rio Grande do Sul-UFRGS (protocolo de pesquisa $n^{\circ} 8 / 10$ ). Os estudantes que aceitaram participar da pesquisa preecheram o Termo de Consentimento Livre e Esclarecido em duas vias (uma para o participante e a outra para o pesquisador).

\section{Resultados}

Foram encontradas correlações positivas entre oito variáveis do Questionário de Memória Autobiográfica (QMA) e a medida de imaginação visual do Questionário Bett's (QBetts). Estes resultados estão descritos na Tabela 2.
Tabela 2

Correlações itens QMA e medida de imaginação visual QBett's

\begin{tabular}{lcc}
\hline \multicolumn{1}{c}{ Itens do QMA } & Visual QBett's & $p$ \\
\hline Revivo o evento & $0,234^{*}$ & 0,017 \\
Ouço o evento & $0,365^{* *}$ & 0,001 \\
Vejo o evento & $0,387^{* *}$ & 0,001 \\
$\begin{array}{l}\text { Lembrança de pessoas falando no } \\
\text { evento }\end{array}$ & $0,321^{* *}$ & 0,001 \\
$\begin{array}{l}\text { Lembrar versus apenas saber que o } \\
\text { evento ocorreu }\end{array}$ & $0,296^{* *}$ & 0,002 \\
$\begin{array}{l}\text { O evento vem em palavras } \\
\begin{array}{l}\text { Viajo de volta ao tempo em que o } \\
\text { evento ocorreu }\end{array}\end{array}$ & $0,281^{* *}$ & 0,004 \\
$\begin{array}{l}\text { O evento vem como uma história } \\
\text { coerente }\end{array}$ & $0,377^{* *}$ & 0,001 \\
\hline$* * p<0,01 * p<0,05$ & & 0,001 \\
\hline
\end{tabular}

Os itens do QMA envolvendo habilidades de recordação acústica e visual foram agrupados ("ouço o evento", "vejo o evento" e "lembro de pessoas falando no evento") e correlacionados com a medida geral de imaginação do QBett's (soma dos escores de imaginação visual e acústica). Foi obtida uma correlação positiva $(r=0,386 ; p=0,001)$.

A variável do QMA “vejo o evento recordado" correlacionou-se negativamente com a proporção de orientações $(r=-0,343 ; p=0,001)$ e positivamente com a proporção de avaliações no relato autobiográfico $(r=0,278 ; p=0,001)$. $\mathrm{O}$ índice de coerência global (escala de 0 a 6 ) correlacionou-se com a variável "evento vem como uma história coerente" e com o número de palavras do relato, sendo esta a maior correlação obtida $(r=0,769 ; p=0,001)$.

Uma análise de variância (ANOVA) foi realizada a fim de verificar diferenças entre cursos universitários quanto às seguintes variáveis: coerência narrativa (orientações, ações e avaliações), coerência global (escala de 0 a 6), número de palavras; escores em estilo visual (QVV), escores em estilo verbal (QVV) e escores de imaginação visual e auditiva (QBett's).

Os escores em estilo verbal foram baseados no agrupamento dos seguintes itens do QVV: perceber-se com maior facilidade com palavras que a maioria; gostar de aprender palavras novas; prazer em trabalhos que requerem uso de palavras; tempo utilizado para aumentar vocabulário; e facilidade em pensar sinônimos para as palavras. A ANOVA revelou diferenças entre os cursos relativas às variáveis: (1) coerência global do relato, $F(3,78)=4,62 ; p=0,005 ;(2)$ número de palavras, $F(3,78)=9,34 ; p=0,001$; e (3) escores em estilo verbal, $F(3,78)=5,45 ; p=0,002$. As médias destas variáveis para cada curso estão relatadas na íntegra na Tabela 3. 
Tabela 3

Médias e desvios das variáveis para os quatro cursos

\begin{tabular}{lccccc}
\hline & $n$ & Média & $D P$ & Mínimo & Máximo \\
\hline Coerência global & & & & & \\
$\quad$ Psicologia & 21 & 3,61 & 1,16 & 2 & 5 \\
Letras & 22 & 3,13 & 1,35 & - & 5 \\
Odontologia & 19 & 2,05 & 1,61 & - & 5 \\
Biomedicina & 20 & 2,50 & 1,60 & - & 6 \\
Total & 82 & 2,85 & 1,53 & - & 6 \\
No de palavras & & & & & \\
Psicologia & 21 & 133,14 & 44,55 & 75 & 244 \\
Letras & 22 & 132,81 & 69,07 & 13 & 279 \\
Odontologia & 19 & 60,21 & 43,66 & 11 & 174 \\
Biomedicina & 20 & 78,10 & 58,27 & 3 & 255 \\
Total & 82 & 102,73 & 63,30 & 3 & 279 \\
Verbal QVV & & & & & \\
Psicologia & 21 & 24,00 & 3,30 & 16 & 29 \\
Letras & 22 & 26,59 & 4,42 & 19 & 33 \\
Odontologia & 19 & 21,89 & 4,21 & 15 & 29 \\
Biomedicina & 20 & 22,55 & 4,28 & 13 & 31 \\
Total & 82 & 23,85 & 4,40 & 13 & 33 \\
\hline
\end{tabular}

Para complementação dos resultados da análise da variância, realizou-se o teste Post hoc Bonferroni que permitiu identificar quais cursos diferem em relação às variáveis investigadas. Quanto à variável coerência, houve diferença entre psicologia e odontologia. Quanto ao número de palavras, houve diferença entre: psicologia e odontologia; psicologia e biomedicina; letras e odontologia; e letras e biomedicina. Quanto aos escores verbais do QVV, identificaram-se diferenças entre letras e odontologia, bem como entre letras e biomedicina. Estes resultados estão descritos na Tabela 4.

Tabela 4

Diferenças entre cursos segundo o teste Post hoc Bonferroni

\begin{tabular}{lllcc}
\hline & & $\begin{array}{c}\text { Diferença } \\
\text { entre as } \\
\text { médias }\end{array}$ & $\begin{array}{c}\text { Nível de } \\
\text { significância }\end{array}$ \\
\hline Coerência & Psicologia & Odontologia & $1,56 * *$ & 0,006 \\
\multicolumn{1}{l}{$\begin{array}{l}\text { Número de } \\
\text { palavras }\end{array}$} & Psicologia & Odontologia & $72,93 * *$ & 0,001 \\
& Letras & Biomedicina & $55,04 *$ & 0,013 \\
& & $\begin{array}{l}\text { Odontologia } \\
\text { Biomedicina }\end{array}$ & $72,60^{* *}$ & 0,000 \\
& & & 0,012 \\
Verbal & Letras & Odontologia & $4,69 * *$ & 0,003 \\
QVV & & Biomedicina & $4,04 *$ & 0,012 \\
\hline$* * p<0,01 * p<0,05$ & & &
\end{tabular}

A partir dos dados obtidos no teste Post hoc, constatou-se não haver diferenças significativas entre psicologia e letras, tampouco entre odontologia e biomedicina. Assim, utilizou-se o teste $t$ de Student a fim de verificar se o grupo de cursos com características mais verbais (psicologia e letras) diferiria do grupo com características menos verbais (odontologia e biomedicina). A análise confirmou diferenças significativas entre os dois grupos (psicologia e letras versus odontologia e biomedicina) quanto à coerência $[\mathrm{t}(80)=$ $3,423 ; p=0,001]$, número de palavras $[\mathrm{t}(80)=5,230 ; p=$ $0,001]$; e escore verbal $[\mathrm{t}(80)=3,377 ; p=0,001]$. Os resultados desta análise encontram-se resumidos na Tabela 5 .

Tabela 5

Teste t para diferenças entre grupo mais verbal (psicologia e letras) versus grupo menos verbal (odontologia e biomedicina)

\begin{tabular}{lcccccc}
\hline & $t$ & Df & $p$ & $\begin{array}{c}\text { Média grupo mais } \\
\text { verbal }\end{array}$ & $\begin{array}{c}\text { Média grupo menos } \\
\text { verbal }\end{array}$ & $\begin{array}{c}\text { Diferença das } \\
\text { médias }\end{array}$ \\
\hline Coerência & 3,423 & 80 & 0,001 & 3,32 & 2,23 & 1,09 \\
N $^{\text {p palavras }}$ & 5,230 & 80 & 0,001 & 130,53 & 66,94 & 63,59 \\
Verbal QVV* & 3,377 & 80 & 0,001 & 25,13 & 22,04 & 3,09 \\
\hline
\end{tabular}

* Questionário Verbalizador-visualizador

Também foram analisadas diferenças entre sexo quanto às diversas variáveis de interesse (índices de coerência narrativa, número de palavras, escore verbal, escore visual, escore de imaginação e processos cognitivos de recordação autobiográfica). O teste $t$ de Student não revelou diferenças estatisticamente significativas.

\section{Discussão}

As expectativas de correlação entre variáveis verbais/ visuais de memória autobiográfica (QMA) e estilo cognitivo verbal/visual (QVV) não foram confirmadas. Este resultado talvez se deva à inconsistência psicométrica do QVV 
quanto à avaliação do estilo cognitivo visual (Alencastro e cols., 2010), impedindo a verificação adequada das possíveis correlações.

A totalidade das correlações se deu entre variáveis de memória autobiográfica, indicadores de coerência narrativa e escores de imaginação visual. As correlações encontradas entre a medida de imaginação visual (Questionário Bett's) e itens tanto visuais quanto verbais do QMA contrariaram a expectativa inicial de que a medida de imaginação visual se correlacionaria apenas com itens visuais do QMA ("vejo o evento" e "lembrança do cenário onde ele ocorreu"). Este resultado apontou para a necessidade de investigar se haveria correlação entre a medida de imaginação geral do Questionário Bett's (soma dos escores de imaginação visual e acústica) e o grupo de itens do QMA envolvendo habilidades de recordação visual (imagens) e acústica (palavras). Essa correlação foi confirmada ( $r=0,386 ; p=0,001)$, o que talvez indique que tanto as habilidades verbais quanto visuais de recordação possuem um fator comum: a habilidade de imaginação. Assim, este resultado sugere que o tipo específico de imaginação (acústica ou visual) não interfere na recordação dos elementos do evento, mas sim na habilidade geral de imaginação. Sem esta habilidade geral, não seria possível evocar tanto elementos verbais quanto visuais de um episódio.

A correlação negativa entre o item "vejo o evento recordado" e as orientações no relato (informações sobre o ambiente físico, pessoas presentes etc.) contradisse a expectativa de que alta lembrança de imagens poderia acarretar alta descrição de informações visuais e contextuais do evento. Por sua vez, a correlação positiva entre esta variável do QMA (vejo) e a proporção de avaliações do relato parece corroborar a hipótese de que as avaliações e o sentido atribuído a um evento determinam a vivacidade de suas imagens. Nesta perspectiva, a consolidação de um evento pessoal na memória dependeria do significado a ele atribuído. Achados semelhantes foram encontrados em estudo com o uso dos mesmos instrumentos e formas de análise (Gauer, 2005). Investigando possíveis correlações entre variáveis do QMA e índices de coerência narrativa (orientações, ações e avaliações), não foram encontradas relações entre vivacidade de imagens recordadas e proporção de orientações no relato autobiográfico. As orientações, por sua vez, correlacionaram-se com a variável do QMA "importância do evento". Este resultado, somado aos achados do presente estudo, sugere uma relação entre lembrança vívida de detalhes contextuais do evento e as avaliações acerca do seu significado.

A correlação positiva observada entre coerência narrativa global (escala de 0 a 6) e a variável de memória autobiográfica "evento recordado como uma história coerente" aponta uma possível semelhança entre a coerência com que o evento é lembrado e a coerência com que ele é narrado em forma escrita. Este resultado corrobora a hipótese de que haveria relação entre representação e expressão de eventos autobiográficos.
O índice de coerência narrativa global correlacionou-se positivamente com o número de palavras do relato. A força e significância estatística desta correlação $(r=0,769 ; p=$ $0,001)$ pode ser explicada de duas maneiras. Uma explicação é que um estilo narrativo coerente indicaria preferência verbal-narrativa, a qual refletiria em maior satisfação no ato de narrar e consequente aumento no volume do relato. A explicação alternativa é que não seria possível estabelecer uma narrativa coerente via um relato com reduzido volume de palavras. Assim, quanto maior a quantidade de texto, maior seria a possibilidade de contemplar os requisitos para uma narrativa coerente - relações temporais, temáticas e avaliativas entre os fatos autobiográficos.

Quanto à identificação de perfis cognitivos e narrativos, as diferenças mais acentuadas foram encontradas entre: (1) psicologia e odontologia quanto às variáveis: índice de coerência e volume de relato (número de palavras); (2) letras e odontologia em relação às variáveis: volume de relato e escore verbal.

Os estudantes de psicologia apresentaram um índice de coerência significativamente maior quando comparado ao curso de odontologia. O mesmo achado se deu quanto ao volume de relato, já que foi comprovada diferença estatisticamente significativa entre os estudantes de psicologia (maior número de palavras) e os de odontologia (menor número de palavras). Esse dado se mostra congruente com a forte correlação encontrada entre volume de relato e coerência narrativa global $(r=0,769 ; p=0,001)$. O escore verbal e volume de relato dos estudantes de letras foram acentuadamente maiores quando comparados aos estudantes de odontologia. Este resultado sugere uma possível relação entre preferência verbal e volume do relato.

Desta forma, as principais diferenças encontradas entre os cursos foram relativas à coerência global, ao número de palavras, e aos escores verbais. Nestes quesitos, porém, verificaram-se semelhanças entre estudantes de psicologia e letras, bem como entre odontologia e biomedicina. $\mathrm{O}$ grupo "mais verbal" (psicologia e letras) apresentou maiores médias quanto ao escore verbal, coerência narrativa e volume do relato.

Estes resultados corroboram estudos anteriores que identificaram em alunos de psicologia um alto interesse por áreas verbais, subjetivas e narrativas (Bueno e cols., 2004; Levenfus, 2002). Não foram encontradas pesquisas que investigassem os perfis cognitivos em estudantes de odontologia e letras. No entanto, a presença de habilidades verbais e narrativas em estudantes de letras é amplamente referida na literatura (Kolb, 1981).

No presente estudo, os alunos de biomedicina apresentaram baixos escores em preferências verbais e habilidades narrativas, o que se refletiu em relatos mais pontuais (menor quantidade de palavras). Assumindo o pressuposto de que o curso de biomedicina guarda semelhanças com o curso de medicina, conclui-se que este resultado é congruente com evidências anteriores de que alunos de medicina possuem 
uma forte predominância de conceituação abstrata com vistas a respostas pontuais e específicas (Sobral, 2005).

Desta forma, os resultados confirmam semelhanças entre: (1) estudantes de psicologia e letras - com maiores médias em quesitos verbais e narrativos, cujo perfil profissional parece relacionar-se a estas habilidades; e (2) entre odontologia e biomedicina - com menores escores em quesitos verbais e narrativos, cujo perfil profissional parece relacionar-se a aspectos conceituais e pontuais, mais distantes das preferências verbais e narrativas. No exercício interpretativo destes dados aventamos a seguinte explicação: no estilo cognitivo do primeiro grupo de estudantes predomina o detalhamento descritivo-narrativo; no estilo cognitivo do segundo grupo predomina um movimento de abstração dos detalhes.

A vertente de teorização de Bruner (1986/1997) acerca das diferenças entre pensamento paradigmático e pensamento narrativo alicerça o seguinte entendimento: os estudantes de biomedicina e odontologia apresentariam um estilo cognitivo com predominância do pensamento paradigmático, voltado ao raciocínio abstrato e formulação de leis gerais. Por sua vez, estudantes de letras e psicologia - comumente incluídos na área humana - apresentariam um estilo cognitivo com predominância do pensamento narrativo, isto é, voltado à construção de narrativas bem encadeadas, detalhadas e envolventes.

A investigação de Graff (2005) sustenta esta interpretação, dada as evidências de que sujeitos com altos escores em estilo cognitivo verbal tendem a explorar situações em partes, preocupando-se com detalhes e com o aprofundamento em um tema, enquanto sujeitos com altos escores visuais veem a estrutura como um todo, preocupando-se mais com os aspectos gerais da situação. Desta forma, podemos compreender os altos escores verbais, o grande volume de relato, e o alto índice de coerência narrativa em estudantes de letras e psicologia como um reflexo da predominância do pensamento narrativo descrito por Bruner.

\section{Considerações finais}

Os principais resultados encontrados podem ser resumidos da seguinte forma: preferências verbais parecem relacionar-se com maior volume de relato autobiográfico, e o volume de relato correlacionou-se fortemente com o índice de coerência narrativa global. Logo, é possível deduzir que estas três variáveis interagem em uma mesma direção. No entanto, as análises correlacionais com o total de participantes não indicaram relação entre escore verbal e volume do relato, tampouco entre escore verbal e coerência narrativa. A relação entre as três variáveis encontrou apoio na forte correlação entre volume de relato e coerência, sobretudo nas análises de diferenças entre psicologia e letras versus odontologia e biomedicina.

Estes achados parecem indicar que o campo das comparações entre cursos se mostra adequado ao estudo de variáveis verbais e narrativas, por considerar mais cuidadosamente o perfil cognitivo de cada profissão. Além disso, as evidências encontradas incentivam a continuidade de pesquisas que investiguem a hipótese dos estilos cognitivos, a fim de clarificar se eles realmente influenciam o aprendizado, a percepção, a recordação de eventos e outras funções cognitivas.

É importante ressaltar que os resultados obtidos não são generalizáveis para populações. Desta forma, as diferenças encontradas não devem ser apressadamente estendidas a outros estudantes que não os do presente grupo. Futuros estudos devem investigar se as diferenças aqui encontradas serão confirmadas.

\section{Referências}

Alencastro, L. S., Piccoli, L. F., \& Gomes, W. B. (2010). A avaliação do estilo cognitivo verbal e visual: Estrutura fatorial do Questionário Verbalizador-visualizador. Avaliação Psicológica, 9(1), 35-41.

Bohn, A., \& Berntsen, D. (2008). Life story development in childhood: The development of life story abilities and the acquisition of cultural life scripts from late middle childhood to adolescence. Developmental Psychology, 44(4), 1135-1147.

Bruner, J. (1997). Realidade mental, mundos possíveis (M. A. G. Domingues, Trad.). Porto Alegre: Artmed. (Original publicado em 1986)

Bruner, J., Goodnow, J., \& Austin, A. (1956). A study of thinking. New York: Wiley.

Bueno, J. M. H., Lemos, C. G., \& Tomé, F. A. M. F. (2004). Interesses profissionais de um grupo de estudantes de psicologia e suas relações com inteligência e personalidade. Psicologia em Estudo, 9(2), 271-278.

Campos, A., \& Pérez-Fabello, M. J. (2005). The Spanish version of Betts' Questionnaire upon mental imagery. Psychological Reports, 96(1), 51-56.

Cegarra, J., \& Hoc, J. M. (2006). Cognitive styles as an explanation of experts individual differences: A case study in computer-assisted troubleshooting diagnosis. International Journal of Human-Computer Studies, 64(2), 123-136.

Conway, M. A. (2001). Phenomenological records and the self-memory system. In C. Hoerl \& T. McCormack (Eds.), Time and memory: Issues in philosophy and psychology (pp. 235-255). Oxford, UK: Oxford University Press.

Cools, E., \& Van den Broeck, H. (2007). Development and validation of the cognitive style indicator. Journal of Psychology: Interdisplinary and Applied, 141(4), 359-387.

Elnick, A. B., Margrett, J. A., Fitzgerald, J. M., \& LabouvieVief, G. (1999). Benchmark memories in adulthood: Central domains and predictors of their frequency. Journal of Adult Development, 6(1), 45-59.

Felder, R. M., \& Spurlin, J. E. (2005). Applications, reliability, and validity of the index of learning styles. International Journal of Engineering Education, 21(1), 103-112. 
Gauer, G. (2005). Memória autobiográfica: Qualidades fenomenais da recordação consciente e propriedades atribuidas a eventos pessoais marcantes. Tese de doutorado não publicada, Universidade Federal do Rio Grande do Sul, Porto Alegre.

Gauer, G., \& Gomes, W. B. (2008). Recordação de eventos pessoais: Memória autobiográfica, consciência e julgamento. Psicologia: Teoria e Pesquisa, 24(4), 507-514.

Graff, M. G. (2005). Individual differences in hypertext browsing strategies. Behaviour \& Information Technology, 24(2), 93-99.

Habermas, T., \& Bluck, S. (2000). Getting a life: The emergence of the life story in adolescence. Psychological Bulletin, 126(5), 748-769.

Jones, C., Reichard, C., \& Mokhtari, K. (2003). Are students' learning styles discipline specific? Community College Journal of Research and Practice, 27(5), 363-375.

Knez, I. (2006). Autobiographical memories for places. Memory, 14(3), 359-377.

Kolb, D. A. (1981). Learning styles and disciplinary differences. In A. W. Chickering (Ed.), The modern American college (pp. 232-255). San Francisco, CA: Jossey-Bass.

Kozhevnikov, M. (2007). Cognitive styles in the context of modern psychology: Toward an integrated framework of cognitive style. Psychological Bulletin, 133(3), 464-481.

Kraemer, D. J., Rosenberg, L. M., \& Thompson-Schill, S. L. (2009). The neural correlates of visual and verbal cognitive styles. Journal of Neuroscience, 29(12), 3792-3798.

Levenfus, R. S. (2002). Levantamento de interesses profissionais. In R. S. Levenfus \& D. H. Soares (Orgs.), Orientação vocacional ocupacional (pp. 209-223). Porto Alegre: Artmed.

Martins, R. M. M., Santos, A. A. A., \& Bariani, I. C. D. (2005). Estilos cognitivos e compreensão leitura em universitários. Paidéia (Ribeirão Preto), 15(30), 57-68.

Massa, L. J., \& Mayer, R. E. (2006). Testing the ATI hypothesis: Should multimedia instruction accommodate verbalizer-visualizer cognitive style? Learning and Individual Differences, 16(4), 321-335.

Mastor, K. A., \& Ismail, A. H. (2004). Personality and cognitive style differences among matriculation engineering and information technology students. World Transactions on Engineering and Technology Education, 3(1), 101-105.

Mayer, R. E., \& Massa, L. J. (2003). Three facets of visual and verbal learners: Cognitive ability, cognitive style, and learning preference. Journal of Educational Psychology, 95(4), 833-846.

Ormrod, J. E. (2008). Educational psychology: Developing learners. Upper Saddle River, NJ: Pearson.

Pashler, H., McDaniel, M., Rohrer, D., \& Bjork, R. (2008). Learning styles: Concepts and evidence. Psychological Science in the Public Interest, 9(3), 105-199.
Pérez-Fabello, M. J., \& Campos, A. (2004). Factor structure and internal consistency of the Spanish version of the Gordon Test of Visual Imagery Control. Psychological Reports, 94(3, Pt 1), 761-766.

Peterson, C., \& Biggs, M. (1998). Stitches and casts: Emotionality and narrative coherence. Narrative Inquiry, $8(1), 51-76$.

Richardson, A. (1977). Verbalizer-visualizer: A cognitive style dimension. Journal of Mental Imagery, 1(1), 109-126.

Rubin, D. C. (1998). Beginnings of a theory of autobiographical remembering. In C. P. Thompson, D. J. Herrmann, D. Bruce, J. D. Read, D. G. Payne, \& M. P. Toglia (Eds.), Autobiographical memory: Theoretical and applied perspectives (pp. 47-67). Mahwah, NJ: Lawrence Erlbaum.

Rubin, D. C. (2005). A basic-systems approach to autobiographical memory. Current Directions in Psychological Science, 14(2), 79-83.

Rubin, D. C., \& Greenberg, D. L. (2003). The role of narrative in recollection: A view from cognitive psychology and neuropsychology. In G. D. Fireman, T. E. McVay, Jr., \& O. J. Flanagan (Eds.), Narrative and consciousness: Literature, psychology, and the brain (pp. 53-85). New York: Oxford University Press.

Rubin, D. C., Schrauf, R. W., \& Greenberg, D. L. (2003). Belief and recollection of autobiographical memories. Memory and Cognition, 31(6), 887-901.

Ruttun, R. (2009). The effects of visual elements and cognitive styles on student's learning in hypermedia environment. World Academy of Science, Engineering and Technology, (49), 963-971.

Santos, M. M. S., \& Gomes, W. B. (1997). Relações entre qualidades e quantidades no estudo da consciência pública e privada [Resumo]. Salão de Iniciação Científica, 10, Feira de Iniciação Científica, 7, 170. Porto Alegre: Ed. UFRGS.

Sheehan, P. W. (1967). A shortened form of Betts' questionnaire upon mental imagery. Journal of Clinical Psychology, 23(3), 386-389.

Sobral, D. T. (2005). Estilo de aprendizagem dos estudantes de medicina e suas implicações. Revista Brasileira de Educação Médica, 29(1), 5-12.

Sternberg, R. J., Grigorenko, E. L., \& Zhang, L. (2008). Styles of learning and thinking matter in instruction and assessment. Perspectives on Psychological Science, 3(6), 486-506.

Westbury, C., \& Dennett, D. C. (2000). Mining the past to construct the future: Memory and belief as forms of knowledge. In D. L. Schacter \& E. Scarry (Eds.), Memory, brain, and belief (pp. 11-32). Cambridge, UK: Harvard University Press.

Westcott, T. B., \& Rosenstock, E. (1976). Reliability of two measures of imagery. Perceptual and Motor Skills, 42(3, Pt. 2), 1037-1038. 
Luciano da Silva Alencastro é doutorando pelo Programa de Pós-graduação em Psicologia da Universidade Federal do Rio Grande do Sul.

Luciano Ferreira Piccoli é graduando em Psicologia pela Universidade Federal do Rio Grande do Sul.

William Barbosa Gomes é Professor Associado do Instituto de Psicologia da Universidade Federal do Rio Grande do Sul.

Recebido: 01/10/2010

$1^{a}$ revisão: $27 / 10 / 2010$

Aceite final: 15/02/2011 